\title{
Avaliação das Principais Propriedades Físicas e Mecânicas da Madeira de Eucalyptus benthamii Maiden et Cambage
}

\author{
Bruna Verediana Müller ${ }^{1}$, Márcio Pereira da Rocha ${ }^{2}$, \\ Alexsandro Bayestorf Cunha ${ }^{3}$, Ricardo Jorge Klitzke², \\ Marcos Felipe Nicoletti ${ }^{3}$
}

${ }^{1}$ Instituto Federal Catarinense - IFC, Rio do Sul/SC, Brasil

${ }^{2}$ Departamento de Engenharia e Tecnologia Florestal - DETF, Universidade Federal do Paraná - UFPR, Curitiba/PR, Brasil ${ }^{3}$ Departamento de Engenharia Florestal, Universidade do Estado de Santa Catarina - UDESC, Lages/SC, Brasil

\begin{abstract}
RESUMO
O presente estudo teve como objetivo determinar as propriedades físicas e mecânicas da madeira de Eucalyptus benthamii Maiden et Cambage. Foram determinadas as seguintes propriedades físicas: massa específica aparente a 12 e $0 \%$ de umidade, massa específica aparente básica e os coeficientes de contração, além das propriedades mecânicas, por meio dos ensaios de flexão estática, compressão paralela às fibras, cisalhamento e dureza, para a idade de seis anos. Com base nos resultados obtidos na determinação das propriedades físicas, a madeira de E. benthamii, na idade estudada, pode ser classificada como moderadamente pesada e dimensionalmente instável. Em relação às propriedades mecânicas, de modo geral, a espécie apresentou baixa resistência, o que possivelmente está relacionado com a grande proporção de madeira juvenil presente no material estudado.
\end{abstract}

Palavras-chave: eucalipto, qualidade da madeira, propriedades tecnológicas.

\section{Evaluation of the Main Physical and Mechanical Properties of Eucalyptus benthamii Maiden et Cambage Wood}

\begin{abstract}
The present study aimed to assess the physical and mechanical properties of Eucalyptus benthamii Maiden et Cambage wood. The following physical properties were determined: apparent density at 12 and $0 \%$ moisture content, basic density, and indices of contraction. Mechanical properties were determined through tests of static bending, compression parallel to the grain, shear, and hardness in six-year-old wood. Based on the results obtained in the determination of physical properties, E. benthamii wood at the age studied can be classified as moderately heavy and dimensionally unstable. The species showed low resistance with respect to mechanical properties, which can be related to the large amount of juvenile wood observed in the material studied.
\end{abstract}

Keywords: eucalyptus, wood quality, technological properties. 


\section{INTRODUÇÃO}

O Eucalyptus benthamii Maiden et Cambage é uma árvore com altura variando de 30 a $45 \mathrm{~m}$ (FAO et al., 2004), pertencente à família Myrtaceae, subfamília Leptospermoideae e que, segundo Higa \& Pereira (2003), embora apresente características distintas, faz parte do mesmo grupo botânico do Eucalyptus viminalis Labill.

Em seu país de origem, a Austrália, a espécie é considerada em risco de extinção, estando a sua distribuição natural restrita ao oeste da cidade de Sydney, em planícies ao longo do Rio Nepean e seus afluentes (Benson \& McDougall, 1998). A espécie ocorre de forma natural em solos férteis, nas partes planas das deposições dos rios, em regiões de altitude inferior a $100 \mathrm{~m}$, com temperatura média máxima de $26^{\circ} \mathrm{C}$ e temperatura média mínima de $4^{\circ} \mathrm{C}$, com ocorrência de geadas leves e precipitação média anual de 1.100 mm (Higa, 1999; Higa \& Pereira, 2003).

A espécie vem apresentando taxas de crescimento elevadas e tem exibido capacidade de crescer em diversos sítios, incluindo aqueles sujeitos a secas e geadas (FAO et al., 2004). No entanto, de acordo com Higa \& Pereira (2003), são escassos os resultados de plantios com a espécie e, além disso, os usos da madeira ainda estão sendo avaliados (Paludzyszyn Filho et al., 2006).

Apesar de existirem poucos estudos sobre as características tecnológicas da madeira de E. benthamii, destaca-se o interesse considerável pela espécie no sul da África e em partes da América do Sul, onde vem sendo difundida na implantação de povoamentos florestais para a produção de celulose e papel (FAO et al., 2004).

No Brasil, a espécie foi introduzida pela Embrapa Florestas em 1988, em Colombo, Estado do Paraná, a partir de dez matrizes procedentes de Wentworth Falls, NSW (Austrália) (Graça et al., 1999). Na Região Sul, esta espécie tem mostrado bom crescimento e resistência a geadas, em plantios experimentais com dois e três anos de idade no Estado de Santa Catarina (Higa, 1999). Já no Paraná, no município de Guarapuava, de acordo com Paludzyszyn Filho et al. (2006), o E. benthamii apresentou-se mais resistente à geada do que o Eucalyptus dunnii, sendo que, na região de Dois Vizinhos, Higa \& Carvalho (1990) observaram, em plantios com idade de 45 meses, sobrevivência de $70 \%$, altura média de 16 m e DAP médio de $15 \mathrm{~cm}$, concluindo que a espécie merece atenção especial dos melhoristas.

Por tudo isso, nos últimos anos, tem se constatado, no sul do Brasil, principalmente em regiões de ocorrência de geadas, uma expansão considerável das áreas de produção de E. benthamii. Contudo, apesar de todos os resultados apresentados pela espécie e do aumento maciço dos seus plantios, os principais trabalhos realizados, até o momento, a respeito da sua utilização estão basicamente relacionados ao seu uso potencial na indústria de celulose e papel.

Assim, para que o E. benthamii seja mais bem aproveitado pela indústria madeireira, é imprescindível se conhecerem, de forma mais precisa, as suas características. Desta forma, o estudo das propriedades físicas e mecânicas da sua madeira se caracteriza como uma importante ferramenta, propiciando a sua correta utilização e contribuindo para a geração de produtos de maior valor agregado.

Com relação às propriedades físicas da madeira sólida a serem avaliadas, aponta-se a massa específica como um dos principais parâmetros de utilização, por se correlacionar diretamente com a maior parte das outras propriedades e também com a composição celular da madeira, expressando sua qualidade final e seu comportamento nos processos tecnológicos e industriais (Oliveira et al., 2005; Batista et al., 2010).

Outra importante variável física é a retratibilidade, responsável pela variação das dimensões das peças de madeira, que ocorrem quando há redução do teor de umidade abaixo do ponto de saturação das fibras (PSF) (Oliveira et al., 2010). O conhecimento da retratibilidade, de acordo com Silva (2002), constitui um importante subsídio para a correta condução do processo de secagem e a utilização final do produto, com o intuito de minimizar a ocorrência de possíveis defeitos na madeira.

Em relação às propriedades mecânicas da madeira, destacam-se, como as mais investigadas: resistência aos esforços de compressão, flexão, tração, cisalhamento e fendilhamento (Scanavaca Junior, 2001), cujo estudo, conforme Araújo (2007), é fundamental para definir adequadamente a 
aplicação da madeira às mais variadas utilizações, especialmente em relação àquelas que requerem uma maior resistência e rigidez.

Diante do exposto, o presente estudo teve como objetivo determinar as propriedades físicas e mecânicas da madeira de Eucalyptus benthamii Maiden et Cambage, tendo em vista que esta espécie está sendo plantada em larga escala na região serrana do Estado de Santa Catarina.

\section{MATERIAL E MÉTODOS}

O material utilizado neste estudo foi coletado em um plantio comercial de E. benthamii com aproximadamente seis anos de idade e espaçamento inicial de 2,5 $\times 2,0 \mathrm{~m}$, submetido a um desbaste seletivo por baixo com $20 \%$ de intensidade. O referido povoamento florestal está localizado no Distrito de São Jorge, Município de Cerro Negro, pertencente à região do Planalto Serrano, Estado de Santa Catarina.

A escolha das árvores se deu de forma visual, considerando-se determinados aspectos, como tronco cilíndrico e baixa incidência de galhos, e selecionando-se oito árvores com DAP (diâmetro à altura do peito) entre 20 e $30 \mathrm{~cm}$. Após a seleção, as árvores foram abatidas e, da sua porção basal, foram retiradas as toras, com $2 \mathrm{~m}$ de comprimento, utilizadas na caracterização das propriedades tecnológicas da madeira sólida. De cada uma das toras selecionadas, foi retirado um semibloco da parte central, com 8 $\mathrm{cm}$ de espessura, contendo medula. Os semiblocos foram processados em vigas de $8 \times 8 \mathrm{~cm}$, retirandose da viga central toda a porção da medula. Os corpos de prova utilizados para a caracterização física e mecânica da espécie foram retirados de cada uma das vigas e colocados em uma câmara climatizada, com temperatura de $20^{\circ} \mathrm{C}$ e umidade relativa de $65 \%$, até atingirem aproximadamente $12 \%$ de umidade de equilíbrio, ou seja, peso constante, com exceção dos corpos de prova para análise das propriedades físicas, que foram colocados em água.

A determinação das propriedades físicas e mecânicas da madeira de E. benthamii foi realizada no Laboratório de Propriedades da Madeira na Universidade do Estado de Santa Catarina, no Município de Lages.
Em relação às propriedades físicas, foram determinadas a massa específica aparente com a madeira climatizada, a massa específica aparente básica e os coeficientes de contração, tendo como base a NBR 7190/97 - Projeto de Estruturas de Madeira. Para tanto, os corpos de prova foram saturados em água, posteriormente colocados em uma sala climatizada, até atingirem aproximadamente $12 \%$ de umidade, e finalmente foram colocados em estufa a $103+/-2^{\circ} \mathrm{C}$ até $0 \%$ de umidade. De posse das massas e das medidas das dimensões lineares nos três pontos mencionados, foram calculadas as variáveis de interesse.

Já as propriedades mecânicas foram determinadas em uma máquina universal de ensaios, tendo como base a norma COPANT 555 (COPANT, 1973) para o cálculo do módulo de ruptura (MOR) e o módulo de elasticidade (MOE) para a flexão estática, e a NBR 7190/97 para os ensaios de compressão, cisalhamento e dureza. Para tanto, foram utilizados 15 corpos de prova para cada ensaio.

\section{RESULTADOS E DISCUSSÃO}

\subsection{Propriedades físicas}

Considerando-se os resultados obtidos para as propriedades físicas (Tabela 1), verificou-se que o valor observado para a massa específica aparente a $0 \%$ para a madeira de $E$. benthamii foi ligeiramente inferior ao valor observado por França \& Cunha (2012), que, ao estudarem a mesma espécie e considerarem a mesma condição de umidade, obtiveram o valor de $0,63 \mathrm{~g} / \mathrm{cm}^{3}$. Contudo, os valores observados para esta espécie estão de acordo com os valores verificados para outras espécies do mesmo gênero. Rocha (2000), avaliando duas espécies de eucalipto, observou os valores de $0,57 \mathrm{~g} / \mathrm{cm}^{3}$ para o E. grandis e $0,77 \mathrm{~g} / \mathrm{cm}^{3}$ para o E. dunnii.

Em relação à massa específica aparente a $12 \%$, o resultado observado para o E. benthamii - do mesmo modo que para a massa específica aparente a $0 \%$ também se apresentou dentro da faixa de valores observada para outras espécies de Eucalyptus. Evangelista et al. (2010), ao estudarem a madeira de E. urophylla com seis anos de idade, observaram o valor médio de $0,55 \mathrm{~g} / \mathrm{cm}^{3}$. Já Arantes (2009), ao avaliar a madeira de um clone de E. grandis 
Tabela 1. Propriedades físicas para a madeira do E. benthamii.

Table 1. Physical properties for E. benthamii wood.

\begin{tabular}{lccc}
\multicolumn{1}{c}{ Propriedade } & Média & Desvio Padrão & $\begin{array}{c}\text { Coeficiente de } \\
\text { Variação (\%) }\end{array}$ \\
\hline Massa específica aparente a $12 \%\left(\mathrm{~g} / \mathrm{cm}^{3}\right)$ & 0,61 & 0,06 & 10,62 \\
\hline Massa específica aparente a $\% \%\left(\mathrm{~g} / \mathrm{cm}^{3}\right)$ & 0,56 & 0,07 & 11,94 \\
\hline Massa específica aparente básica $\left(\mathrm{g} / \mathrm{cm}^{3}\right)$ & 0,52 & 0,04 & 8,22 \\
\hline Contração longitudinal (\%) & 0,53 & 0,15 & 29,92 \\
\hline Contração radial (\%) & 5,91 & 1,49 & 25,19 \\
Contração tangencial (\%) & 13,87 & 5,49 & 35,59 \\
\hline Contração volumétrica(\%) & 18,52 & 6,64 & 27,28 \\
\hline Fator anisotrópico & 2,36 & 0,64 & \\
\hline
\end{tabular}

$\times$ E. urophylla, também com seis anos de idade, considerando três classes diamétricas, verificou valores entre 0,52 e $0,59 \mathrm{~g} / \mathrm{cm}^{3}$.

Note-se que o valor médio de massa específica aparente básica verificado neste estudo para o E. benthamii foi ligeiramente superior aos valores observados por outros autores para a mesma espécie. Pereira et al. (2000), estudando a madeira de $E$. benthamii de diferentes idades, verificaram, aos quatro anos de idade, a densidade básica média da árvore de $0,40 \mathrm{~g} / \mathrm{cm}^{3}$, enquanto que, aos sete anos, o valor observado foi de $0,47 \mathrm{~g} / \mathrm{cm}^{3}$.

Quando comparada a outras espécies do gênero Eucalyptus, a massa específica básica do E. benthamii mostrou-se superior à demonstrada pelo E. grandis $\left(0,45 \mathrm{~g} / \mathrm{cm}^{3}\right)$ (Batista et al., 2010) e inferior à observada para o E. citriodora $\left(0,73 \mathrm{~g} / \mathrm{cm}^{3}\right)$ (Oliveira et al., 2005), podendo assim ser classificada como mediana.

Em relação aos resultados obtidos para contração da madeira de E. benthamii (Tabela 1), os valores verificados aproximaram-se dos observados por Higa \& Pereira (2003), que, ao estudarem os usos potencias desta mesma espécie, verificaram os valores médios de $0,3 \%, 6,9 \%, 13,7 \%$ e 19,5\% para as contrações longitudinal, radial, tangencial e volumétrica, respectivamente, além do fator anisotrópico, cujo resultado obtido foi igual a 2,0.

Sobre os coeficientes de variação obtidos para os resultados de contração (Tabela 1), os mesmos apresentaram-se bastante elevados, demonstrando a heterogeneidade do lenho da espécie estudada, o que compromete diretamente a sua utilização nas aplicações que exigem uma maior estabilidade dimensional.

Quando comparada a outras espécies do gênero Eucalyptus, a contração longitudinal verificada para o E. benthamii mostrou-se elevada. Silveira et al. (1999), ao avaliarem o efeito do espaçamento sobre a variação dimensional de 12 clones híbridos naturais do gênero Eucalyptus, obtiveram, para a contração axial, resultados compreendidos entre 0,29 e 0,31\%. Possivelmente, esta excessiva contração longitudinal observada para o E. benthamii esteja relacionada à presença de madeira juvenil, principalmente quando é considerada a idade do material estudado.

Quanto à contração radial, pôde-se verificar que os valores observados para E. benthamii aproximaram-se dos observados para outras espécies de eucalipto. Batista (2009), estudando a secagem convencional de clones de três espécies de eucalipto, observou valores entre 4,51 e 5,79\% para o E. saligna, entre 4,37 e $4,67 \%$ para o E. grandis e entre 4,98 e $6,59 \%$ para o E. dunnii. Todavia, quando considerada a contração radial apresentada por madeiras tropicais utilizadas comercialmente, os valores observados pelo E. benthamii são consideravelmente elevados.

Em relação ao índice de contração tangencial, verifica-se que o valor médio observado neste estudo está de acordo com os resultados obtidos por Oliveira et al. (2010), que, avaliando a contração da madeira de sete espécies de eucaliptos com sete anos de idade, encontraram valores entre 9,3 (E. cloeziana) e $15,5 \%$ (E. paniculata).

Ao se avaliar a contração volumétrica, verificase que o resultado obtido para o E. benthamii está dentro dos limites observados para outras espécies 
do mesmo gênero. Gonçalves et al. (2009a), avaliando um híbrido clonal de E. urophylla $\times$ E. grandis de diferentes idades e tratamentos silviculturais, observaram índices de contração entre 15,24 e $21,76 \%$. No entanto, apesar de se aproximar dos valores observados para outras espécies de Eucalyptus, a contração volumétrica observada para o E. benthamii é muito elevada, o que se torna mais evidente quando considerados os valores apresentados por espécies utilizadas na produção de serrados (Alves et al., 2012).

Esta elevada contração volumétrica pode dificultar muito a utilização industrial do E. benthamii, sobretudo no que diz respeito ao processo de secagem da madeira, considerando-se a grande possibilidade de formação de defeitos. Além disso, estes defeitos podem ser ainda mais acentuados em função da grã irregular apresentada por esta espécie, que, conhecidamente, tende a reduzir a resistência mecânica e aumentar a ocorrência de deformações de secagem.

A respeito do fator anisotrópico da madeira de E. benthamii, o valor médio obtido se aproxima do valor observado para outras espécies de Eucalyptus (Silva \& Oliveira, 2003; Lopes, 2007; Oliveira et al., 2010), sendo que, de acordo com Oliveira et al. (2010), o fator de anisotropia pode variar de 1,3 a 1,4 para madeiras muito estáveis a mais de 3 para espécies extremamente instáveis dimensionalmente, como ocorre para muitas espécies de Eucalyptus, principalmente quando se trata de árvores de rápido crescimento.

\subsection{Propriedades mecânicas}

Os resultados obtidos para as propriedades mecânicas da madeira de E. benthamii estão apresentados na Tabela 2, na qual pode ser observado que, quanto à resistência à compressão paralela às fibras, os resultados obtidos foram consideravelmente inferiores aos apresentados pela ABNT (1997) na NBR 7190, para 17 espécies de eucaliptos, cuja resistência variou entre 40,3 $\mathrm{MPa}$ (E. grandis) e 78,5 MPa (E. punctata), e o módulo de elasticidade, entre $12.813 \mathrm{MPa}$ (E. grandis) e 19.881 MPa (E. paniculata). Essa menor resistência do $E$. benthamii à compressão paralela às fibras está possivelmente relacionada com as características do material utilizado nos ensaios, o qual, em função da idade, apresentava grande quantidade de madeira juvenil, o que, normalmente, tende a afetar negativamente as propriedades físicas e mecânicas da madeira.

Contudo, pôde-se verificar, também, baixa resistência aos esforços de compressão paralela às fibras em materiais que apresentam maior percentual de madeira adulta. Lima \& Garcia (2010), por exemplo, ao avaliarem a madeira de E. grandis aos 21 anos de idade, verificaram valores entre 35,23 e 61,09 MPa.

Em relação à flexão estática, verifica-se que os resultados obtidos estão de acordo com os observados para outras espécies do gênero Eucalyptus, cujos ensaios foram realizados com material de densidade e idade próximas ao material utilizado neste estudo.

Tabela 2. Propriedades mecânicas para o E. benthamii obtidas a $12 \%$ de umidade.

Table 2. Mechanical properties for E. benthamii obtained at $12 \%$ moisture.

\begin{tabular}{llrrr}
\multicolumn{2}{c}{ Propriedade } & Média & Desvio Padrão & $\begin{array}{c}\text { Coeficiente de } \\
\text { Variação (\%) }\end{array}$ \\
Compressão paralela às fibras & $\mathrm{f}_{\mathrm{c} 0}(\mathrm{MPa})$ & 37,34 & 7,83 & 20,96 \\
& $\mathrm{E}_{\mathrm{c} 0}(\mathrm{MPa})$ & 2565,00 & 735,00 & 28,66 \\
Flexão Estática & $\mathrm{f}_{\mathrm{M}}(\mathrm{MPa})$ & 83,53 & 17,86 & 21,37 \\
Cisalhamento & $\mathrm{E}_{\mathrm{M} 0}(\mathrm{MPa})$ & 9754,67 & 2805,68 & 28,76 \\
\multirow{2}{*}{ Dureza Janka } & $\mathrm{f}_{\mathrm{v}}(\mathrm{MPa})$ & 11,41 & 1,93 & 16,89 \\
& $\mathrm{f}_{\mathrm{H} 0}(\mathrm{~N})$ & 5632,94 & 1432,75 & 25,44 \\
\hline & $\mathrm{f}_{\mathrm{H} \text { Radial }}(\mathrm{N})$ & 5137,70 & 2077,05 & 40,42 \\
\hline
\end{tabular}

$\mathrm{f}_{c 0}=$ resistência à compressão paralela às fibras; $\mathrm{E}_{\mathrm{co}}=$ módulo de elasticidade obtido no ensaio de compressão paralela às fibras;

$\mathrm{f}_{\mathrm{M}}=$ resistência à flexão estática; $\mathrm{E}_{\mathrm{M} 0}=$ módulo de elasticidade obtido no ensaio de flexão estática; $\mathrm{f}_{\mathrm{v}}=$ resistência ao cisalhamento;

$\mathrm{f}_{\mathrm{H} 0}=$ dureza da madeira na direção paralela às fibras obtida pelo método Janka; $\mathrm{f}_{\mathrm{H} \text { Radial }}=$ dureza da madeira na direção radial obtida pelo método Janka; $\mathrm{f}_{\mathrm{H} \text { Tangencial }}=$ dureza da madeira na direção tangencial obtida pelo método Janka. 
Gonçalves et al. (2009b), ao avaliarem a madeira da primeira tora de um híbrido clonal de E. urophylla $\times$ E. grandis, aos 70 meses de idade, provenientes de talhadia simples e reforma, observaram resistência à flexão estática variando de 82,95 a 83,21 MPa, e módulo de elasticidade entre 9.652 e $9.798 \mathrm{MPa}$.

Para a resistência ao cisalhamento, os valores observados foram superiores aos obtidos por Lobão et al. (2004), que, para o E. grandis, verificaram a resistência de $7 \mathrm{MPa}$. Todavia, resultados superiores são observados para outras espécies do gênero Eucalyptus. Rodrigues (2002), ao estudar E. grandis, E. saligna e E. citriodora, observou, como menor valor característico para a resistência ao cisalhamento, 7,94 $\mathrm{MPa}$, sendo que as demais avaliações apresentaram resultados variando entre 8,71 e 15,92 MPa. Neste mesmo sentido, Scanavaca Junior \& Garcia (2004) observaram valores ainda mais elevados ao estudarem o E. urophylla, verificando, como resultado médio, 18,64 MPa.

Em relação à dureza Janka, os resultados obtidos para as três faces da madeira foram inferiores ao observado por Caixeta et al. (2003), que, ao avaliarem 44 genótipos de eucaliptos, observaram, como valor médio para a dureza, $8.178,75 \mathrm{~N}$. Resultados inferiores a estes foram verificados por Lima et al. (2004), que, ao associarem as tensões de crescimento em eucaliptos com outras propriedades, observaram resultados médios entre 5.420 a $7.267 \mathrm{~N}$. Já Gonçalez et al. (2006), ao estudarem a dureza longitudinal e a dureza média para as faces radiais e tangenciais, verificaram para o E. grandis os valores de $5.207,33 \mathrm{~N}$ e $6.511,62 \mathrm{~N}$, respectivamente; observe-se que, para o E. cloeziana, os valores observados foram de $11.110,93 \mathrm{~N}$ e $11.444,36 \mathrm{~N}$.

De modo geral, a madeira de E. benthamii apresentou baixa resistência, não sendo apropriado o seu emprego em usos estruturais pesados. Entretanto, em aplicações menos exigentes em relação à resistência aos esforços de compressão e flexão, o E. benthamii pode ser indicado como matéria-prima complementar na construção civil, sendo indicado para aplicações internas, entre outras utilizações.

\section{CONCLUSÕES}

Considerando-se os resultados obtidos para a massa específica aparente, pode-se classificar a madeira de E. benthamii como moderadamente pesada.
Quanto à contração e ao coeficiente de anisotropia da madeira, os resultados verificados para a madeira de E. benthamii podem ser considerados elevados, indicando elevada instabilidade dimensional e grande propensão da madeira à formação de rachaduras e empenos.

Sobre as propriedades mecânicas, de maneira geral, a madeira de E. benthamii apresentou baixa resistência, não devendo ser recomendada para usos estruturais.

De acordo com os resultados obtidos, recomendase o estudo da espécie com idades mais avançadas, visando a minimizar o efeito da madeira juvenil.

\section{AGRADECIMENTOS}

À Empresa Floko Florestadora Koeche Ltda., pela doação da madeira utilizada neste estudo.

\section{STATUS DA SUBMISSÃO}

Recebido: 10 jul., 2013

Aceito: 28 jul., 2014

Publicado: 21 nov., 2014

\section{AUTOR(ES) PARA CORRESPONDÊNCIA}

\section{Bruna Verediana Müller}

Instituto Federal Catarinense - IFC, Campus Rio do Sul, CEP 89163-356, Rio do Sul, SC, Brasil e-mail: bruna.muller@ifc-riodosul.edu.br

\section{REFERÊNCIAS}

Alves CS, Pereira KRM, Silva TTS, Frota VB, Souza IAT. Avaliação das propriedades físicas de oito espécies usadas em serrarias de Rio Branco, Acre. In: Anais do IV Congresso Florestal Paranaense; 2012; Curitiba. Curitiba; 2012.

Arantes MDC. Variação nas características da madeira e do carvão de um clone de Eucalyptus grandis W. Hill ex Maiden $x$ Eucalyptus urophylla S. T. Blake [tese]. Lavras: Universidade Federal de Lavras; 2009.

Araújo HJB. Relações funcionais entre propriedades físicas e mecânicas de madeiras tropicais brasileiras. Floresta 2007; 37(3): 399-416. http://dx.doi.org/10.5380/ rf.v37i3.9937. 
Associação Brasileira de Normas Técnicas - ABNT. NBR - 7190: Projeto de estruturas de madeira. Rio de Janeiro: ABNT; 1997.

Batista DC. Qualidade da secagem convencional conjunta da madeira de nove clones do gênero Eucalyptus [dissertação]. Curitiba: Universidade Federal do Paraná; 2009.

Batista DC, Klitzke JR, Santos CVT. Densidade básica e retratibilidade da madeira de clones de três espécies de Eucalyptus. Ciência Florestal 2010; 20(4): 665-674.

Benson D, McDougall L. Ecology of Sydney plant species: part 6 Dicotyledon family Myrtaceae. Cunninghamia 1998; 5(4): 808-807.

Caixeta RP, Trugilho PF, Rosado SCS, Lima JT. Propriedades e classificação da madeira aplicada à seleção de genótipos de Eucalyptus. Árvore 2003; 27(1): 23-51.

Comision Panamericana de Normas Tecnicas COPANT. COPANT 555 - Maderas: Método de ensayo de flexion estática. Buenos Aires: COPANT; 1973.

Evangelista WV, Silva JC, Lucia RMD, Lobo LM, Souza MOA. Propriedades físico-mecânicas da madeira de Eucalyptus urophylla S. T. Blake no sentido radial e longitudinal. Ciência da Madeira 2010; 1(2): 1-19. http://dx.doi.org/10.12953/2177-6830.v01n02a01.

Food and Agriculture Organization of the United Nations - FAO. Forest \& Landscape Denmark - FLD. International Plant Genetic Resources Institute - IPGRI. Forest genetic resources conservation and management. Rome: International Plant Genetic Resources Institute; 2004.

França MC, Cunha AB. Determinação da relação resistência/rigidez e teor de umidade da madeira de Eucalyptus benthamii Maiden et Cambage. In: Anais do IV Congresso Florestal Paranaense; 2012; Curitiba. Curitiba; 2012.

Gonçalez JC, Breda LCS, Barros JFM, Macedo DG, Costa GJAF, Vale AT. Características tecnológicas das madeiras de Eucalyptus grandis W. Hill ex Maiden e Eucalyptus cloeziana F. Muell visando o seu aproveitamento na indústria moveleira. Ciência Florestal 2006; 16(3): 329-341.

Gonçalves FG, Oliveira JTS, Lucia RMD, Nappo ME, Sartório RC. Densidade básica e variação dimensional de um híbrido clonal de Eucalyptus urophylla $\mathrm{x}$ Eucalyptus grandis. Árvore 2009a; 33(2): 277-288. http://dx.doi.org/10.1590/S0100-67622009000200009.

Gonçalves FG, Oliveira JTS, Lucia RMD, Sartório RC. Estudo de algumas propriedade mecânicas da madeira de um híbrido clonal de Eucalyptus urophylla x Eucalyptus grandis. Árvore 2009b; 33(3): 501-509. http://dx.doi.org/10.1590/S0100-67622009000300012.

Graça MEC, Shimizu JY, Tavares FR. Capacidade de rebrota e enraizamento de Eucalyptus benthamii. Boletim de Pesquisa Florestal 1999; 39: 135-138.
Higa RCV. Aspectos ecológicos e silviculturais do Eucalyptus benthamii Maiden et Cambage. Boletim de Pesquisa Florestal 1999; 38: 121-123.

Higa AR, Carvalho PER. Sobrevivência e crescimento de doze espécies de eucalipto em Dois Vizinhos, Paraná. In: Anais do VI Congresso Florestal Brasileiro; 1990; Campos do Jordão. São Paulo; 1990. p. 459.

Higa RCV, Pereira JCD. Usos potenciais do Eucalyptus benthamii Maiden et Cambage. Colombo: Embrapa Florestas; 2003.

Lima IL, Garcia JN. Variação da densidade aparente e resistência a compressão paralela às fibras em função da intensidade de desbaste, adubação e posição radial em Eucalyptus grandis Hill Ex-Maiden. Árvore 2010; 34(3): 551-559. http://dx.doi.org/10.1590/S010067622010000300019 .

Lima JT, Trugilho PF, Rosado SCS, Cruz CR. Deformações residuais longitudinais decorrentes de tensões de crescimento em eucaliptos e suas associações com outras propriedades. Árvore 2004; 28(1): 107-116. http://dx.doi.org/10.1590/S0100-67622004000100014.

Lobão SM, Lúcia RMD, Moreira MSS, Gomes A. Caracterização das propriedades físico-mecânicas da madeira de eucalipto com diferentes densidades. Árvore 2004; 28(6): 889-894

Lopes CSD. Caracterização da madeira de três espécies de eucalipto para uso em movelaria [dissertação]. Piracicaba: Universidade de São Paulo; 2007.

Oliveira JTS, Hellmeister JC, Tomazello Filho M. Variação do teor de umidade e da densidade básica na madeira de sete espécies de eucalipto. Árvore 2005; 29(1): 115-127. http://dx.doi.org/10.1590/S010067622005000100013 .

Oliveira JTS, Tomazello Filho M, Fiedler NC. Avaliação da retratibilidade da madeira de sete espécies de Eucalyptus. Árvore 2010; 34(5): 929-936. http://dx.doi. org/10.1590/S0100-67622010000500018.

Paludzyszyn Filho E, Santos PET, Ferreira CA. Eucaliptos indicados para plantio no Estado do Paraná. Colombo: Embrapa Florestas; 2006.

Pereira JCD, Sturion JA, Higa AR, Higa RCV, Shimizu JY. Características da madeira de algumas espécies de eucalipto plantadas no Brasil. Colombo: Embrapa Florestas; 2000.

Rocha MP. Eucalyptus grandis Hill ex Maiden e Eucalyptus dunnii Maiden como Fontes de Matéria Prima para Serrarias [tese]. Curitiba: Universidade Federal do Paraná; 2000.

Rodrigues RAD. Variabilidade de propriedades físicomecânicas em lotes de madeira serrada de eucalipto para a construção civil [dissertação]. Piracicaba: Universidade de São Paulo; 2002. 
Scanavaca Junior L. Caracterização silvicultural, botânica e tecnológica do Eucalyptus urophylla S. T. Blake e de seu potencial para a utilização em serraria [dissertação]. Piracicaba: Universidade de São Paulo; 2001.

Scanavaca Junior L, Garcia JN. Determinação das propriedades físicas e mecânicas da madeira de Eucalyptus urophylla. Scientia Florestalis 2004; (65): 120-129.

Silva JC. Caracterização da madeira de Eucalyptus grandis Hill ex. Maiden, de diferentes idades, visando a sua utilização na indústria moveleira [tese]. Curitiba: Universidade Federal do Paraná; 2002.

Silva JC, Oliveira JTS. Avaliação das propriedades higroscópicas da madeira de Eucalyptus saligna Sm. em diferentes condições de umidade relativa do ar. Árvore 2003; 27(2): 233-239.

Silveira V, Rosado SCS, Trugilho PF, Carvalho D, Martins SC. Variação dimensional da madeira de clones de Eucalyptus cultivados em diferentes espaçamentos. Cerne 1999; 5(2): 105-124. 